\title{
Editorial
}

Im Jahr 2015 beging die Christian-Albrechts-Universität zu Kiel (CAU) ihr 350-jähriges Jubiläum, das mit zahlreichen Ausstellungen und Veranstaltungen gefeiert wurde. Einen wissenschaftlichen Meilenstein stellten sicher die Veröffentlichung der Festschrift „Christian-Albrechts-Universität zu Kiel. 350 Jahre Wirken in Stadt, Land und Welt“ ${ }^{\text {1 }}$ sowie der Launch des Kieler Gelehrtenverzeichnisses - „Kieler Professorinnen und Professoren von 1919 bis 1965“ (KGV) dar. ${ }^{2}$ Während sich erstere der langen Traditionsgeschichte der Christiana Albertina seit 1665 widmete, fokussiert sich das KGV - der digitale Professorenkatalog der CAU - auf die Darstellung der wissenschaftlichen Werdegänge der Kieler Professorenschaft vornehmlich des 20. Jahrhunderts.

\section{Historie des Kieler Gelehrtenverzeichnisses}

Biographische Verzeichnisse in Form von analogen Professorenkatalogen bildeten an vielen Hochschulen von jeher eine wichtige Komponente der Identitätsstiftung und Selbstvergewisserung der eigenen Alma Mater. Das erhobene Datenmaterial vermittelt über die Biographien des akademischen Personals, die klassischerweise anhand der wichtigsten Lebensdaten, akademischen Stationen und Publikationen dargestellt werden, bedeutende Aspekte der Geschichte einer Hochschule. Da die prestigereichen analogen Professorenkataloge aber auf Dauer für die moderne (Universitäts-)Forschung zu wenige Informationen bieten, die darüber hinaus nicht mit weiteren Wissensbestände vernetzt und für Akteurinnen und Akteure einer interessierten Zivilgesellschaft nur schwer zugänglich sind, wurden insbesondere in den letzten zehn Jahren verstärkt Projekte zur Erstellung digitaler Professorenkataloge gefördert. So wurden seit Mai 2010 die bio-bibliographischen Daten aller Kieler Hochschullehrerinnen und Hochschullehrer, die zwischen 1919 und 1965 an der CAU lehrten, von der Abteilung für Regionalgeschichte des Historischen Seminars gesammelt und für die Online-Präsenz systematisch aufgearbeitet. Gemeinsam mit der Arbeitsgruppe Kommunikationssysteme des Instituts für Informatik entstand eine auf dem Semantic Web basierende Datensammlung, die es ermöglicht, überregionale Inhalte zu Kieler Professorinnen und Professoren mit weiteren Ressourcen global zu verknüpfen. Wissenschaftlerinnen und Wissenschaftlern sowie der interessierten Öffentlichkeit stehen nun bereits seit fünf Jahren Informationen zu den wissenschaftlichen Profilen und gesellschaftlichen Aktivitäten von über 1.000 Kieler Hochschullehrerinnen und Hochschullehrern online zur freien Verfügung. Das präsentierte Resultat stieß seither ausweislich der Pressemitteilungen, Frequenz der Besucherinnen und Besucher bei Vor- 
tragsveranstaltungen und Anfragen bzw. Kommentaren seitens Dritter auf eine beeindruckende öffentliche Resonanz und wurde im Jahr 2017 von der Hermann Ehlers Stiftung e.V. mit dem Kai-Uwe von Hassel-Förderpreis prämiert. Es ist von daher bestimmt nicht übertrieben, wenn man das KGV als Flaggschiff der Digital Humanities an der CAU charakterisiert. Die Universitätsbibliothek und das Rechenzentrum der CAU unterstützen das Projekt als langjährige, verlässliche Kooperationspartner. Innerhalb des Projektes wird die interdisziplinäre Zusammenarbeit zwischen Geschichtswissenschaft und Informatik stetig erfolgreich erprobt.

Das KGV ist dabei in den beiden angestammten Bereichen der universitären Hochschule - der Forschung und der Lehre - fest verankert und wirkt darüber hinaus im Sinne der ebenso zentralen und immer wichtiger werdenden ,dritten akademischen Mission“ aktiv in die Gesellschaft hinein.

\section{Das KGV als innovatives Forschungsfeld}

Das KGV wurde frühzeitig in die Lehre des Historischen Seminars implementiert, um junge Geschichtsstudierende mit dem Projekt in Kontakt zu bringen..$^{\underline{3}}$ Durch den Umgang mit einem innovativen Forschungswerkzeug wie dem KGV erwerben diese Kompetenzen im Bereich der digitalen Geschichtswissenschaft. Dieses Feld wird - was die aktuelle Corona-Krise anschaulich und hautnah vor Augen führt - zu einem immer zentraler werdenden Teil der gesamten Geschichtswissenschaft in Forschung und Vermittlung. Digitale Methoden sind längst fester Bestandteil des wissenschaftlichen Arbeitens innerhalb der Fachdisziplin geworden. Dabei spiegeln computergestützte (Such-)Verfahren, digitale Archivierungslösungen sowie Möglichkeiten zur Wissensmodellierung, Datenauswertung, -analyse und -visualisierung nur einen Bruchteil vorstellbarer Instrumente und Methoden digitaler historischer Forschung wider. Auch die theoretische Untersuchung der methodischen Grundlagen digitaler Praktiken, deren Chancen aber auch Grenzen sowie die konkrete Einbindung in die geisteswissenschaftliche Lehre sind Bestandteil der Diskurse geschichtswissenschaftlicher Forschung im 21. Jahrhundert. Die zunehmende Internationalisierung und damit einhergehende standortübergreifende Forschung stellen zudem neue Anforderungen an eine gemeinschaftliche vernetzte Wissensorganisation der Geschichtswissenschaften sowie an den freien Zugang zu Forschungsdaten und -ergebnissen. Auch die Fachkommunikation selber wird dabei in vielen Bereichen digitalisiert. Dazu zählt die Verwendung und Entwicklung geeigneter kollaborativer Werkzeuge, Datenformate sowie Beschreibungsstandards, und es entstehen zunehmend Bedarfe, die in gleicher oder ähnlicher Form den Ansprüchen zeitgerechter Forschung im Allgemeinen ent- 
sprechen. So unterschiedlich die disziplinspezifischen Forschungsdaten auch sein mögen, ist beispielsweise die Reproduktion von Forschungsergebnissen, der dauerhafte Zugang zu Forschungsdaten sowie die Referenzierung dieser eine fachübergreifende Herausforderung im Forschungsdatenmanagement (FDM) zahlreicher, wenn nicht sogar aller wissenschaftlicher Disziplinen. Die Forderung nach einer offenen Wissenschaft (Open Science) und dem damit verbundenen FDM ist daher seit 2019 auch im rechtsverbindlichen Kodex („Leitlinien zur Sicherung guter wissenschaftlicher Praxis“) für Förderanträge bei der Deutschen Forschungsgemeinschaft (DFG) abgebildet. Dieser sieht u. a. einen öffentlichen Zugang zu Forschungsergebnissen und den zugrunde liegenden Forschungsdaten, aber auch die Archivierung für einen angemessenen Zeitraum vor. Ebenso präzisiert die in Cambridge gegründete Open Knowledge Foundation (OKF) $)^{4}$ in ihrer „Offen-Definition“ $\underline{\underline{5}}$ die Bedeutung von „offen“ in Bezug auf Wissen, wobei sie offene Formate und Lizenzen, Maschinenlesbarkeit und den gemeinfreien Zugang als wichtige Aspekte identifiziert und die Anforderungen an diese genauer beschreibt. Die offene Datenbereitstellung und -vernetzung mittels der technischen Möglichkeiten des Semantic Web zeigt sich hierbei als effektives Mittel zur Umsetzung der Anforderungen und vereinfacht die nicht ortsgebundene Recherche. Das KGV bietet als Sammlung strukturierter Personendaten mit seinen Visualisierungs- und Analysemöglichkeiten insbesondere Forscherinnen und Forschern, aber auch der interessierten Zivilgesellschaft vielfältige Informationen zu den Personenprofilen, darüber hinaus aber auch die Möglichkeit zu deren (computergestützter) Weiterverarbeitung. Die gesamte Datenbank, die eine Kollektion von Forschungsdaten und zugleich ein Forschungsergebnis darstellt, ist in einem offenen und strukturierten Format zur freien Nachnutzung als (Linked) Open Data verfügbar, wobei Normdatenverknüpfung sowie einfache Provenienzangaben kontextuale Anknüpfungspunkte bieten. Für die Personeneinträge der Kieler Professorinnen und Professoren wurden dabei im Besonderen Informationen aus der vielfältigen Quellenüberlieferung des Universitätsarchivs der CAU genutzt. ${ }^{-}$Diese wurden im Sinne des Semantic Web erfasst und aufbereitet. Ergänzt werden diese Informationen durch die Verknüpfung mit weiteren Onlineangeboten wie der Gemeinsamen Normdatei (GND) oder Wikidata. ${ }^{?}$ Somit steht den Forscherinnen und Forschern ein quellenbasiertes und gleichzeitig vernetztes Angebot zur Verfügung, mit dem zahlreiche Fragestellungen der Kultur-, Sozialund Bildungsgeschichte untersucht werden können. $\stackrel{\underline{8}}{-}$

Nun bietet die Verknüpfung mit externen digitalen Angeboten, so unterschiedlich diese auch inhaltlich und methodisch ausgestaltet sind, zwar eine enorme Vereinfachung der Recherche, gleichwohl zeigte sich schon früh in der Projektarbeit der Bedarf nach einer technisch einheitlichen Basis und eines deutschlandweit koordinierten Wissensangebots zur professoralen Universitätsgeschichte. 2012 wurde daher die uni- 
versitätsübergreifende Arbeitsgemeinschaft Deutscher Professorenkatalog ins Leben gerufen. Von den damaligen Projektpartnern in Kiel, Leipzig, Rostock, Bamberg und Helmstedt war seinerzeit angedacht, lokal erfasstes Datenmaterial zu den deutschen Professoren vom 16. bis zum 20. Jahrhundert gebündelt für überregionale Recherchearbeiten und Fragestellungen auswertbar und zugänglich zu machen.

Konkretisiert werden diese Bestrebungen aktuell durch die Antragstellung zu einem Deutschen Digitalen Gelehrtenverzeichnis (DDG) an der Akademie der Wissenschaften und der Literatur Mainz. Hierfür haben die Universitäten Kiel, Marburg und Mainz ein gemeinsames Projekt im Forschungsprogramm der deutschen Akademien der Wissenschaften beantragt, das quellenbasierte, zitierfähige und möglichst vollständige Informationen zu den ordentlichen und außerordentlichen Professorinnen und Professoren erheben will, die im Zeitraum von 1871 bis 1961 an den 27 deutschen Volluniversitäten und 15 Technischen Hochschulen bzw. Universitäten gewirkt haben. Die erstmalige Katalogisierung dieser Personengruppe vom Kaiserreich bis zur jungen Bundesrepublik und Deutschen Demokratischen Republik würde grundlegende allgemeine Erkenntnisse zum Wandel des Wissenschaftsbetriebs je nach politischem System sowie zu Generierung und Transfer von Wissen in die Gesellschaft zulassen.

Das großangelegte Langzeitvorhaben wird aktuell durch zwei Projektanträge der Abteilung für Regionalgeschichte bei der Deutschen Forschungsgemeinschaft (DFG) flankiert, die auf die vergleichende Erforschung der NS-Reichsuniversitäten während des Zweiten Weltkrieges und der Landesuniversitäten Kiel, Greifswald, Marburg und Tübingen im sogenannten langen 19. Jahrhundert abzielen. Letzteres Vorhaben ist wiederum Teil eines Verbundantrages, an dem auch die landesgeschichtlichen Lehrstühle der Universitäten Heidelberg, Trier und Tübingen beteiligt sind.

\section{Grenzenlose Forschung - Open Access am KGV}

Als Teil der offenen Wissenschaft lässt sich auch die Open Access-Bewegung betrachten, ${ }^{9}$ die den freien bzw. offenen Zugang zu wissenschaftlicher Literatur fordert, um Publikationsstrukturen, die eine Privatisierung des von der Allgemeinheit finanzierten Wissens bewirken, entgegenzutreten. Publikationen als Ergebnis öffentlich geförderter Forschung sollten der Idee nach der Gesellschaft grundsätzlich kostenfrei zur Verfügung gestellt werden. Ebenso ist intendiert, die Distribution wissenschaftlicher Erkenntnisse sowie ihre Sichtbarkeit und Verfügbarkeit zu sublimieren. Mit ihrer ,Strategie 2020` für Open Access bekennt und fördert die schleswig-holsteinische Landesregierung den offenen digitalen Zugang zu den Ergebnissen wissenschaftlicher Forschung und den Quellen des kulturellen Erbes..$^{10}$ In Zusammenarbeit zwischen dem 
Land Schleswig-Holstein, den Hochschulen und den dort tätigen Wissenschaftlerinnen und Wissenschaftlern soll Open Access zum bevorzugten Publikationsmodell werden. $\stackrel{11}{ }$ Für das Veröffentlichen im Open Access haben sich in den vergangenen Jahrzehnten zwei Wege etabliert: der Goldene und der Grüne Weg. Während der Goldene Weg die Open Access-Erstveröffentlichung eines wissenschaftlichen Textes beschreibt, so wie es beim vorliegenden ersten Band der Kieler Studien zur Universitätsund Wissenschaftsgeschichte (KSUW) der Fall ist, bezeichnet der Grüne Weg die parallele oder nachträgliche Open Access-Zweitveröffentlichung, z.B. über private oder institutionelle Webseiten, oder institutionelle Publikationsserver bzw. Repositorien. Wichtig ist, dass gemäß der „Berlin Declaration on Open Access to Knowledge in the Sciences and Humanities“ aus dem Jahr 2003는 Open Access nicht nur bedeutet, wissenschaftliche Erkenntnisse frei verfügbar, sondern auch frei nachnutzbar zu machen, durch eine möglichst offene urheberrechtliche Lizenzierung. $\frac{13}{}$

Dadurch, dass das KGV bereits früh in der Lehre verankert wurde, konnten in den letzten Jahren immer mehr Studierende erfolgreich dazu motiviert werden, ihre aussichtsreichen Bachelor- oder Masterarbeiten im Forschungsumfeld des KGV zu verfassen, so dass junge und innovative Zugänge zum Thema generiert werden konnten. Mit der neuen Publikationsreihe KSUW werden nun erstmals einige Ergebnisse dieser ausgezeichneten und spannenden Abschlussarbeiten allen Interessierten im Sinne von Open Access und Open Science niedrigschwellig zugänglich gemacht.

\section{Abseits der Universität? Skandal, Terrorismus, Kriegsgefangenschaft in der Kieler Universitätsgeschichte des 20. Jahrhunderts}

Die erste Ausgabe der ab sofort in lockerer Folge erscheinenden KSUW leistet einen Beitrag zur Universitätsgeschichtsschreibung für das 20. Jahrhundert. Mit dem Aufsatz von Lisa Bittner begeben wir uns in das Kiel der unmittelbaren Nachkriegszeit. Bittner beleuchtet in ihrem Beitrag „Wir warten auf Prof. Brandt - Ein Kriegsgefangenenschicksal aus Kiel“ die letztlich erfolglosen Versuche verschiedener Akteurinnen und Akteure, den Kieler Juraprofessor Hans Kurt Paul Brandt aus der jugoslawischen Kriegsgefangenschaft zu befreien. Der Beitrag fragt nach den Motivationen der einzelner Gruppen, sich für dessen Heimkehr einzusetzen, und hinterfragt unter anderem das starke Engagement der Studierenden, die dieser Thematik einen eigenen Artikel im Kieler Studenten, der ersten Studierendenzeitung der CAU nach dem Zweiten Weltkrieg, widmeten. Gleichfalls legt der Beitrag professorale Netzwerke offen, die während der NS-Zeit geknüpft wurden und noch lange darüber hinauswirkten. Diesen gelang es nicht nur, die junge Studierendenschaft für ihre Zwecke einzuspannen, son- 
dern auch, sich gegenseitig in der jungen Bundesrepublik zahlreiche Karrierechancen zu ermöglichen.

2017 rüttelte ein Thema die gesamte westliche Welt auf: Die „\#MeToo'Debatte legte das bis dato weitgehend tabuisierte Ausmaß sexueller Belästigung und sexueller Übergriffe an Frauen in nicht gekannter Weise offen. Dass gerade die jahrhundertealte Ordinarienuniversität, die erst durch die Hochschulreformen der späten 1960er und frühen 1970er Jahre umgestaltet wurde, mit ihren klaren Hierarchiestrukturen und ihrer mangelnden Transparenz einen Nährboden für derlei Übergriffe bot, die kaum geahndet wurden, zeigt der Beitrag von Karen Bruhn und Swantje Piotrowski. Anhand ausgewählter Fallbeispiele illustrieren die Autorinnen, wie die CAU innerhalb der letzten 120 Jahre auf Vorwürfe sexueller Nötigung gegen ihre Studenten und Professoren reagierte und wie mit Frauen als Zeuginnen oder Opfern in solchen Verfahren umgegangen wurde.

Chronologisch daran anschließend, ist der nachfolgende Beitrag in der Zeit der Hochschulreformen angesiedelt, genauer gesagt im bewegten Jahr 1968. Ende der 1960er Jahre sah sich Professor Dr. Horst Gärtner mit einer Verleumdungskampagne der Hamburger Zeitschrift konkret konfrontiert, die ihn beschuldigte, im Rahmen einer Forschungsstelle der Bundeswehr an ABC-Waffen experimentiert zu haben. Welche Auswirkungen diese Anschuldigungen auf das weitere Leben und den Ruf des Kieler Professors hatten und welche Ausmaße die Proteste gegen ihn annahmen, erfährt man im Beitrag „Wenn die konkret dreimal klingelt - Die Horst Gärtner-Affäre“ von Nele Dittrich. Darin begegnet den Leserinnen und Lesern eine mittlerweile stark politisierte Studierendenschaft, die im Gegensatz zu jener in Lisa Bittners Beitrag weder vor lautstarken Protestaktionen noch vor juristischen Auseinandersetzungen zurückschreckte, um Professor Gärtner und sein Institut zu diffamieren und ihre Forderungen nach einer Demokratisierung der Universität durchzusetzen.

Schließlich widmet sich Swantje Piotrowski einer besonders turbulenten Phase in der bundesdeutschen Geschichte, deren Konsequenzen auch vor den Toren der altehrwürdigen Christiana Albertina keinen Halt machten: Mit ihrem - im Übrigen preisgekrönten - Beitrag „Kieler Professoren, ihre Söhne und der linke Terrorismus - Zum Umgang mit akademischem Traditionsbewusstsein und politischer Radikalisierung an der CAU“ verfolgt die langjährige Mitarbeiterin des KGV das Schicksal der Professorensöhne Georg von Rauch und Thomas Weisbecker, die zunächst in der Studentenbewegung der 1960er Jahre aktiv waren, sich danach der linksradikalen militanten Szene in West-Berlin anschlossen und schließlich beide einen gewaltsamen Tod fanden. Im Fokus des Beitrages Piotrowskis steht im Besonderen, wie die Institution der Universität auf das Schicksal der beiden jungen Männer reagierte und sich zu den linksterroristischen Gruppierungen in Deutschland positionierte. 
Der vorgelegte Band gibt einen tieferen Einblick in ein bislang vernachlässigtes Segment der jüngeren Geschichte der Universität und thematisiert dabei nicht nur die Lebenswege von Professorinnen und Professoren, die naturgemäß im Fokus des KGV stehen, sondern liefert auch wichtige Erkenntnisse zu den Kieler Studierenden, die in den einzelnen Beiträgen als zunehmend politisierende Akteursgruppe zutage treten. Während diese in den 1950ern im Fall Kurt Brandts anscheinend noch diskussionslos bereit waren, personelle Belange der NS-belasteten Professorenschaft zu unterstützen, tritt die Studierendenschaft der 1960er als selbstbewusster Part einer gesellschaftskritischen politischen Bewegung auf, wobei sie sich allerdings in der Gärtner-Affäre abermals für die politischen Zwecke eines Dritten einspannen ließen - diesmal für die Regimekritik eines linken Blattes.

In ihrem Auftaktband beleuchten die KSUW, wie die Christiana Albertina sich zu verschiedenen gesellschaftlichen und politischen Phänomenen und Skandalen des 20. Jahrhunderts verhalten und positioniert hat und zeigen somit exemplarisch, wie stark ausgeprägt die Wechselwirkungen zwischen Universität und Gesellschaft zu allen Zeiten waren und sind.

Oliver Auge, Karen Bruhn und Thorge Petersen

Prof. Dr. Oliver Auge ist Direktor der Abteilung für Regionalgeschichte mit dem Schwerpunkt zur Geschichte Schleswig-Holsteins in Mittelalter und Früher Neuzeit am Historischen Seminar der CAU.

Kontakt:oauge@email.uni-kiel.de

Karen Bruhn, M.A., ist Wissenschaftliche Mitarbeiterin im Projekt Kieler Gelehrtenverzeichnis der Abteilung für Regionalgeschichte am Historischen Seminar der CAU. Kontakt:k.bruhn@email.uni-kiel.de

Thorge Petersen, B.Sc., studiert Informatik an der CAU und ist Mitarbeiter im Projekt Kieler Gelehrtenverzeichnis der Abteilung für Regionalgeschichte am Historischen Seminar der CAU. Kontakt:petersen@rz.uni-kiel.de 


\section{Anmerkungen}

Der Beitrag ist unter der Creative-Commons-Lizenz Namensnennung 4.0 international veröffentlicht. Den Vertragstext finden Sie unter: https://creativecommons.org/licenses/by/4.0/deed.de. Bitte beachten Sie, dass einzelne, entsprechend gekennzeichnete Teile des Werks von der genannten Lizenz ausgenommen sein bzw. anderen urheberrechtlichen Bedingungen unterliegen können.

1 Oliver Auge (Hg.), Christian-Albrechts-Universität zu Kiel. 350 Jahre Wirken in Stadt, Land und Welt, Kiel/Hamburg 2015, abrufbar unter: https://nbn-resolving.org/urn:nbn:de:gbv:8-publ-10890 (21.08.2020, 10:16 Uhr).

2 https://cau.gelehrtenverzeichnis.de (14.08.2020, 20:05 Uhr).

$\underline{3}$ Beispielsweise das Projektseminar im WS 2018/19 "Studierende schaffen Wissen - Die Kieler Vorlesungsverzeichnisse als Quelle zur Untersuchung der Wissenschaftstransformation" angeboten und geleitet von Karen Bruhn und Dr. Swantje Piotrowski oder das Aufbauseminar im WS 2017/18 „Was sind eigentlich Digital Humanities? Zur neuen digitalen Methodik in den Geistes- und Kulturwissenschaften am Beispiel eines geschichtswissenschaftlichen Forschungsprojektes" angeboten und geleitet von Dr. Swantje Piotrowski.

4 Gemeinnützige Stiftung und starker Akteur der Open Data-Bewegung, welche sich zum Ziel gesetzt hat, offenes Wissen zu erzeugen sowie als Standard zu verbreiten. Vgl. Open Knowledge Foundation, Mission, abrufbar unter: https://okfn.org/about/ (15.08.2020, 11:00 Uhr).

$\underline{5}$ Vgl. Open Definition Version 2.1, abrufbar unter: https://opendefinition.org/od/2.1/en/ (15.08.2020, 11:00 Uhr).

$\underline{6}$ An vorderster Stelle sind hierbei die Personalakten der Abteilungen 47 (Universitätsarchiv) sowie 811 (Kultusministerium) zu nennen. Diese wurden durch die systematische Auswertungen der Entnazifizierungsverfahren (Abteilung $460 \mathrm{LASH}$ ) und der Hochschullehrerkartei der NSDAP (Bundesarchiv Berlin Lichterfelde) ergänzt.

I Freie und gemeinsam erstellte Datenbank zur Speicherung strukturierter Daten zur Unterstützung der Projekte der Wikimedia Commons. Vgl. Wikidata, Introduction, abrufbar unter: https://www.wikidata.org/wiki/Wikidata:Introduction (15.08.2020, 11:00 Uhr).

8 Vgl. Margrit Prussat, Vernetzte Biographien. Die Medizinische Fakultät im Bamberger Professorinnen- und Professorenkatalog, in: Eine Wissenschaft im Umbruch. Andreas Röschlaub (1768-1835) und die deutsche Medizin um 1800, hg. von Mark Häberlein u. Margrit Prussat (Bamberger historische Studien/18), Bamberg 2018, S. 237-259.

9 Die Vorläufer der Open Access Bewegung entstanden bereits mit den Preprint- und Dissertationsservern der 1990er Jahre. Vgl. Margo Bargheer, Saskia Bellem und Birgit Schmidt, Open Access und Institutional Repositories - Rechtliche Rahmenbedingungen, in: Rechtliche Rahmenbedingungen von Open Access-Publikationen, hg. von Gerald Spindler (Göttinger Schriften zur Internetforschung/2), Göttingen 2006, S. 1-21, hier S. 4, abrufbar unter: http://dx.doi.org/10.17875/gup2006-115 (09.04.2020, 15:31 Uhr).

10 Vgl. Ministerium für Soziales, Gesundheit, Wissenschaft und Gleichstellung des Landes Schleswig-Holstein, Strategie 2020 der Landesregierung Schleswig-Holstein für Open Access, abrufbar unter: https://oa-info.sh/wp-content/ uploads/2019/12/141118 msgwg OpenAccessStrategie.pdf (14.08.2020, 17:45 Uhr).

11 Vgl. Ministerium für Bildung, Wissenschaft und Kultur des Landes Schleswig-Holstein, Open Access in Schleswig-Holstein, abrufbar unter: https://oa-info.sh/open-access-in-schleswig-holstein/ (09.04.2020, 15:45 Uhr).

$12 \mathrm{Vgl}$. Berlin Declaration on Open Access to Knowledge in the Sciences and Humanities, abrufbar unter: https://openaccess. mpg.de/67605/berlin declaration engl.pdf (09.04.2020, 15:05 Uhr).

13 Vgl. Bargheer/Bellem/Schmidt, Open Access (wie Anm. 9), S. 6. 